\title{
Hulfeld, Stefan, Theatergeschichtsschreibung als kulturelle Praxis. Wie Wissen über Theater entsteht
}

\section{Laure Gauthier}

\section{OpenEdition}

Journals

Édition électronique

URL : http://journals.openedition.org/ifha/1593

DOI : $10.4000 /$ ifha. 1593

ISSN : 2198-8943

Éditeur

IFRA - Institut franco-allemand (sciences historiques et sociales)

Référence électronique

Laure Gauthier, « Hulfeld, Stefan, Theatergeschichtsschreibung als kulturelle Praxis. Wie Wissen über

Theater entsteht », Revue de I'IFHA [En ligne], Date de recension, mis en ligne le 01 janvier 2008,

consulté le 22 septembre 2020. URL : http://journals.openedition.org/ifha/1593 ; DOI : https://doi.org/ 10.4000/ifha.1593

Ce document a été généré automatiquement le 22 septembre 2020.

(C)IFHA 


\title{
Hulfeld, Stefan,
}

\section{Theatergeschichtsschreibung als kulturelle Praxis. Wie Wissen über Theater entsteht}

\author{
Laure Gauthier
}

Il manquait aux études théâtrales une étude épistémologique retraçant les grandes étapes de la constitution d'une historiographie du théâtre depuis le XVIe s. jusqu'à nos jours : c'est le pari ambitieux que relève S.H. qui retrace les grandes étapes au cours desquelles l'historiographie du théâtre s'est constituée. S.H. ne cherche pas seulement à écrire l'histoire d'une discipline universitaire, il entend aussi permettre à l'historiographie contemporaine du théatre de se renouveler en prenant conscience de ce qu'elle a été.

L'auteur, s'inscrivant dans une perspective d'histoire culturelle, postule que l'historiographie du théâtre est une pratique culturelle. Le théâtre est conçu non dans sa dimension théorique et a-historique, mais comme un art en évolution, soumis à une histoire. S.H. étudie les conditions dans lesquelles est né en Europe un savoir spécifique au théâtre et les intentions poursuivies par ceux qui ont écrit une histoire du théâtre. Il met en lumière le lien entre la constitution d'un discours spécifique et la société dans lequel celui-ci est apparu. S.H. démontre que les historiens du théâtre ont tenté à la fois d'agir sur la pratique du théâtre et sur la société.

L'étude diachronique est structurée en quatre volets : dans une première partie, l'auteur se consacre à l'étude des étapes préalables à la naissance d'une historiographie du théâtre. Il étudie les premières formes de textes consacrés au drame aux XVIe et XVIIe s., notamment ceux écrits par des nobles assistant à des représentations dans les villes-étapes de leur Grand Tour, les poétiques élaborées pour la plupart en référence à celle d'Aristote et enfin le discours " religieux ", issu de la Réforme ou de la ContreRéforme, qui envisage l'histoire de la production théâtrale en fonction de critères moraux. Ces étapes ont permis, selon S.H., la constitution d'une véritable 
historiographie qui prend symboliquement naissance au siècle suivant avec les Réflexions historiques et critiques sur les différens théâtres de l'Europe de Luigi Riccoboni. S.H. montre comment, au XVIIIe s., l'historiographie du théâtre européen s'est paradoxalement constituée autour d'un discours sur la nation visant à la sacralisation de sa propre tradition et l'exclusion des productions étrangères : son apparition va de pair avec celle de l'historiographie des Lumières. Elle était fondée sur la foi en un progrès des arts à l'image du processus de civilisation de l'humanité et sur la hiérarchisation des cultures et des répertoires. C'est au dépassement de cette historiographie normative des Lumières tel qu'il est opéré à partir de la fin du XIXe s. avec l'apparition d'une nouvelle conception de l'histoire, l'historicisme, que S.H. consacre sa troisième partie. Le XXe s., quant à lui, est marqué par la volonté des historiographes du théâtre, influencés notamment par l'historicisme et l'école des Annales, de consolider les fondements scientifiques de leur objet d'étude. S.H. étudie le rôle clé que joua Max Hermann, fondateur en 1919 du premier institut de recherche théâtrale. Il évoque par ailleurs les travaux de deux chercheurs qui ont, selon lui, marqué le plus la recherche sur l'histoire du théâtre au XXe s. : ceux de Ludovico Zorzi, qui définit le principe historiographique de " théâtre et ville » (" il teatro e la città »), fondé sur l'étude des lieux du théatre et les interactions entre leurs dimensions artistiques et sociales; et ceux de Rudolph Münz qui reconceptualisa la notion de " théâtralité ", élargissant ainsi le champ des études théâtrales et y faisant entrer par exemple l'« autre théâtre ", le théâtre des troupes ambulantes. Dans la quatrième et dernière partie, l'auteur dresse un bilan à partir d'une confrontation des concepts dialectiques de "pratique » et de "théorie ». Il constate combien le rapport entre le discours sur le théâtre et la pratique du théâtre est problématique et il esquisse de nouvelles perspectives pour l'historiographie contemporaine du théâtre, fondé sur un rapport équilibré entre ces deux domaines.

La monographie de S.H. est la première à établir l'histoire de l'historiographie du théâtre. L'auteur illustre de façon tout à fait convaincante le lien indissociable entre l'historiographie et l'historiographie théâtrale ; il montre combien la conception qu'a une époque du théâtre est tributaire d'une conception de l'histoire. Il est à mettre à son crédit d'avoir voulu embrasser une période allant de la première modernité à nos jours et de faire apparaître combien la production d'un siècle influence celle d'un autre. Il évoque par ailleurs l'importance de l'histoire du savoir et de l'histoire des concepts pour l'historiographie du théâtre et évoque à chaque époque des concepts clés, mettant en lumière, par exemple, l'importance de la réception de l'aristotélisme aux XVIe et XVIIe s., la pensée des philosophes des Lumières notamment de Voltaire, ou encore l'historicisme. Mais la faiblesse de l'ouvrage réside précisément dans cet entrecroisement d'histoire sociale et d'histoire des concepts. L'auteur montre très hâtivement l'influence de concepts majeurs de l'historiographie sur l'écriture d'histoires du théâtre : comment expliquer le silence qui est fait sur l'influence de Hegel ou de l'historiographie romantique qui a apporté une nouvelle façon d'envisager l'histoire du théâtre (cf. la revalorisation du baroque par les frères Schlegel) ou encore comment apprécier le choix qui est fait d'éluder Nietzsche et la Kulturkritik qui influença les historiens du théâtre et les avant-gardes ; pour le XXe s., seules les œuvres de trois historiens sont traitées. Le rôle de Walter Benjamin et de l'École de Francfort est trop hâtivement abordé, tout comme les réflexions de Philippe Lacoue-Labarthe et de Jean-Luc Nancy sont ignorées ; quant à la recherche anglo-saxonne, elle est à peine évoquée. Si l'auteur a eu le mérite de vouloir retracer l'histoire de l'historiographie du 
théâtre depuis le XVIe s., le caractère lacunaire et arbitraire des choix dits les plus représentatifs n'en ressort pas moins pour la partie consacrée aux XIXe et XXe s. Par ailleurs, on peut regretter la faiblesse de l'introduction qui est trop brève (4 pages) : l'auteur n'y définit pas le théâtre qu'il envisage (ce n'est qu'en cours d'analyse qu'il indique qu'il n'entend pas faire l'étude des pratiques théâtrales ni traiter des cérémonies, du théâtre chanté ou du théâtre de marionnettes...) ; il n'y précise pas non plus sa conception de l'histoire du savoir, pas plus qu'il n'y définit le lien entre l'histoire du savoir et des concepts et l'histoire sociale qui est au cœur même de sa méthode. Cet essai, très documenté, stimulant et original, demeure une œuvre pionnière dans l'historiographie du théâtre, mais demande à être complété.

Laure Gauthier (Université de Reims - Champagne-Ardenne) 